\title{
The Internal State Mediating Between Decision-Making and Arousal
}

\author{
Ryo Yoneda \\ Shizuoka University \\ Shizuoka, Japan \\ yoneda.ryo.17@shizuoka.ac.jp
}

\author{
Junya Morita \\ Shizuoka University \\ Shizuoka, Japan \\ j-morita@inf.shizuoka.ac.jp
}

\begin{abstract}
To improve cognitive performance with the support of computational systems such as virtual agents, it is necessary to examine changes in decision-making caused by emotions. Many studies have examined the relationship between emotions and decisionmaking by manipulating external factors. However, most of these studies only present the stimuli that evoke emotions and do not consider the internal characteristics of the participants. Therefore, it is necessary to consider the interactions between the internal and external factors. Based on the dual-process model of decisionmaking, the fast process (System 1) and the slow process (System 2) were considered as internal factors of the decision-makers. To examine the effect of each internal factor, two experiments were conducted,where participants completed a gambling task, in which multimodal external factors, such as the lightning color as a visual stimulus, the background music (BGM) as an auditory stimulus, and the facial expressions of agents as a social stimulus, were manipulated. In Experiment 1, the participants' processes were assumed to be under System 1, as their attention was reduced in crowdsourcing experiments. In Experiment 2, the state of the participants was assumed to be under System 2 by implementing additional rewards, depending on the score and questions for rule confirmation. Consequently, the arousal level evoked by stimuli was found to have a significant impact on decision-making in Experiment 1 than in Experiment 2. The indices of decision-making that varied with stimuli were also different between the two experiments. Thus, the distinction between the two processes intermediates the effects of emotional arousal on decision-making.
\end{abstract}

\section{CCS CONCEPTS}

- Human-centered computing $\rightarrow$ Empirical studies in ubiquitous and mobile computing.

\section{KEYWORDS}

emotion, decision-making, arousal, dual-process model

\section{ACM Reference Format:}

Ryo Yoneda and Junya Morita. 2021. The Internal State Mediating Between Decision-Making and Arousal. In Proceedings of the 9th International Conference on Human-Agent Interaction (HAI '21), November 9-11, 2021, Virtual

Permission to make digital or hard copies of part or all of this work for personal or classroom use is granted without fee provided that copies are not made or distributed for profit or commercial advantage and that copies bear this notice and the full citation on the first page. Copyrights for third-party components of this work must be honored

For all other uses, contact the owner/author(s).

HAI '21, November 9-11, 2021, Virtual Event, Japan

(c) 2021 Copyright held by the owner/author(s).

ACM ISBN 978-1-4503-8620-3/21/11.

https://doi.org/10.1145/3472307.3484179
Event, Japan. ACM, New York, NY, USA, 9 pages. https://doi.org/10.1145/ 3472307.3484179

\section{INTRODUCTION}

The research field of human-agent interaction (HAI) includes decision support as a major topic. As suggested by studies in the recent conference (e.g., [28,31]), decision-making is often influenced by the emotional states. Understanding the mechanisms of emotional influence in decision-making is crucial for ascertaining agent-based support leading to rational decision-making.

Experimental studies have also shown evidence of difficulty in controlling emotions independently, without external support (e.g., [17]). To overcome this difficulty, in addition to the agent-based support $[28,31]$, techniques for self-regulation, such as mindfulness, have so far been developed [18]. However, we cannot use the methods discussed so far (direct advise from an agent or self-regulation techniques) while conducting other tasks simultaneously, as human attention has limitations in allocating multiple activities. Therefore, controlling decision-making influenced by emotions in action remains a challenge.

Considering such limitations, we adopted the surrounding environment as a method of regulating emotions that influence decisionmaking. For example, ambient light, sound, and facial expressions of agents around us can be considered influential factors of the change in emotion. Research has been conducted on implicit biofeedback, manipulating such external environmental factors [6, 23, 33, 37]. However, such studies do not consider the internal factors that distinguish the basic cognitive processes behind decision-making. There is a continuous dissuasion of the internal states that affect decision-making $[5,10,13,19,29]$. Among these theories, Kahneman's theory states that humans use two different systems when making decisions: System 1 and System 2 [19]. System 1 works automatically and rapidly, producing impressions and intuitions and requires no effort. System 2 assigns attention to difficult intellectual activities that require deliberate thinking, such as complex calculations; thus, it requires significant attention. Although the details are debatable $[10,29]$, the distinction of attention levels on a task has been accepted in a broad research community.

Therefore, if we support decision-making from the viewpoint of emotion, it is necessary to consider the interactions between internal and external factors. Nevertheless, the effect of stimuli in different internal states on, an axis of emotional state [30], and decision-making are affected by stimuli in different internal states. In this study, we investigated the effects of multimodal stimuli in manipulating external factors on emotions and decision-making. 


\section{RELATED WORK}

In this section, we review previous studies related to emotion, decision-making, and the support of decision-making, while focusing on emotional factors.

First, emotions are triggered by many input modalities. Sotos et al. confirmed that the beats per minute (BPM) and note value of music evokes emotions: a BPM ranging from 120 to 90 evokes sadness, while whole notes to the eighth notes evoke high arousal [14]. The relationship between color and emotion has also been studied. There is a positive correlation between color wavelength and arousal [21, 34]. Furthermore, it was found that the positive and negative emotions of group work members were contagious to other members [2]. Additionally, the above study found that this contagion can change the attitudes toward cooperation, eventually influencing individual performance.

Such emotional fluctuations are said to influence decision-making. Damasio reported that patients whose the ventral medial prefrontal cortex, a region of the brain associated with emotion, is damaged were unable to make appropriate decisions, despite having no cognitive problems [8]. He proposed a somatic marker hypothesis based on this case [9]. The somatic marker hypothesis states that somatic response signals influence rational decision-making significantly. Based on Damasio's findings, detailed studies have been conducted to validate the relationship between emotions and decision-making. For example, Ohira examined the relationship between exploration in decision-making and arousal [27]. The results showed that the exploration during decision-making increased when the participants had high sympathetic arousal. Additionally, this study suggests that the role of arousal may differ depending on the task.

Moreover, interactive systems have also been developed to influence emotions and decision-making. For example, cooperative agents affect work efficiency, calmness, and comfort [35]. In this experiment, anthropomorphic agents that perform actions, such as facial expressions were used. A system that transformed the users' own facial expressions on the display, was further developed [37]. The experiment conducted using the system showed that there were changes in emotions. It also influenced preferences for options through the facial expression feedback. However, this requires the users' attention and can only be used in situations where a user can see their own expression. Some systems use implicit biofeedback to change the users' emotions and decision-making. Costa found that haptic feedback, which is calculated based on heartbeats, influences anxiety and performance [6]. There is also a system that reduces the subject's breathing rate by feeding the user's breathing back as fluctuations in the BGM [23]. Thus, the influence of the external environment on emotions and decision-making has been studied. However, most of these experiments did not consider the internal state of the subject. Decision-making changes depending on the state in the dual-process model [13]. Therefore, it is necessary to conduct experiments under which these are considered.

Based on the above theories of emotion and decision making $[8,9,19,27]$, we conducted two experiments to examine how stimuli affect decision-making in situations involving the two processes in the dual-process model. By conducting these experiments, we examined the role of arousal in decision-making in different internal states.

\section{TASK AND ASSUMPTIONS}

To examine the relationship between arousal and decision-making, we conducted the Wisconsin Card Sorting Test (WCST) [12], which is designed to create a reversal learning, and to evaluate the functions of the prefrontal cortex [17]. Apparently, this task is related to working memory $[15,20]$. Additionally, System 1 in the dualprocess model does not require working memory, whereas System 2 uses working memory [26]. Furthermore, arousal levels affect the cognitive functions of the brain, including working memory $[1,25,32]$. Therefore, we thought it would be appropriate to conduct experiments using this task.

There are several versions of this task. In this study, we constructed an original task environment following several studies $[20,22,24]$. Figure 1 presents the play screen of the task environment, which includes four displayed cards and 64 fixed-sequence response cards. Each of the displayed and the response cards shows figures whose attributes include form (circles, rectangles, triangles, or crosses), color (red, blue, green, or yellow), and number (one, two, three, or four). The displayed cards, which comprise one red circle, two blue rectangles, three green triangles and four yellow crosses (from left to right), are always placed in front of the participant (see Figure 1). The participants classified the response cards in their hand, which were handled randomly under the displayed cards that they considered to be in the same category. The three possible sorting categories were form, color, and number. One of the three categories was the correct category at each point of the task. However, the participants could not know it. After classifying a card, the participant was given feedback on whether the answer was correct or not. If the answer was correct, the effect of coins piling up was triggered to let them know the correct answer on the screen. One hundred points were further added to their score. If the answer was incorrect, the coins disappear, and 100 points were deducted from the score. Furthermore, if the correct answer was given five times in a row, the correct category was changed without warning. In this game, this was repeated for 30 turns. In the task environment, participants could access a document describing the above rules freely, and at any time, by pressing the button located at the upper right corner of the screen.

In this task environment, we manipulated environmental factors assumed to have an effect on arousal and decision-making, as summarized as below.

- Assumption 1: Effects of environmental factors on arousal - Effect of color stimuli on arousal: Red lighting induces high arousal while blue lighting induces low arousal [21, 34].

- Effect of sound stimuli on arousal: A high-BPM of BGM induces high arousal, while the low-BPM of BGM induce low arousal [14].

- Effect of emotional contagion on arousal: Smiley faces induce high arousal among participants, while sad faces evoke low arousal [2].

- Assumption 2: Variation in decision-making by arousal

- Speed of decision-making: Environmental factors that induce high arousal reduce the number of time participants spend on the task compared with environmental factors that induce low arousal. 
- Deliberation of decision-making: Environmental factors that induce low arousal also lead to the adoption of more deliberate behavior by participants compared with environmental factors that induce high arousal.

- Performance of decision-making: Environmental factors that induce low arousal also lead to more beneficial decisionmaking than environmental factors that induce high arousal. Since this indicator is the final output of the task, it is also expected to be influenced by deliberation and speed.

In this study, these assumptions about arousal and decisionmaking were tested in two experiments in which the participants were assumed to have different internal states. Considering the dual-process model of decision making [19], we can hypothesize that the effect of arousal and variations in decision-making appear differently at the different deliberation levels (Systems 1 and 2). For example, it might be plausible that fast and careless decision-makers are significantly influenced by the environment, while deliberate and careful decision-makers are relatively independent of the environment. In the following experiments, we will test this hypothesis by examining how these two systems affect the two assumptions.

\section{EXPERIMENT 1: ENVIRONMENTAL FACTORS AFFECTING SYSTEM 1}

Experiment 1 is aimed at ascertaining how the above assumptions are observed in System 1's dominant situation.

\subsection{Method}

4.1.1 Design. The participants included 265 people recruited from Lancers, a Japanese crowdsourcing service. The average age of the participants was 41.9 years old. Of these, 166 were male, and 99 were female. The participants were paid 100 yen (approximately 1 US dollar) as a show-up fee. Furthermore, it is difficult to manipulate the internal state of the participants directly. Therefore, we manipulated the arousal level. It has been pointed out that the participants' attention to the experiment diminished in crowdsourcing experiments relative to face-to-face experiments [16]. The manipulated arousal leads to a change in the internal state. Therefore, it can be assumed that they engaged in a task that entailed low arousal, namely System 1 .

To examine the effects of each stimulus on their arousal and decision-making, we adopted a 3-way factorial design: 2 (illumination: red vs. blue conditions) $\times 2$ (BGM: high-BPM vs. low-BPM conditions) $\times 2$ (emotional contagion: smile vs. sad conditions). In the red illumination condition, the background color was set to 255 , 0, 0 in RGB format, and the blue illumination was set to $0,0,255$ in RGB format. The high-BPM and low-BPM conditions were set to 150 and 80 BPM, respectively. ${ }^{1}$ Emotional contagion was controlled by the facial expressions of the icons in the background during the execution of the game. Figure 1 shows that the task interfaces varied according to illumination and emotion contagion factors. The participants were assigned to the experimental condition in the order of access to the experimental system in order to ensure that each group had equal numbers of participants. ${ }^{2}$

\footnotetext{
${ }^{1}$ Both conditions used the same audio loop, "Dry Disco Breaks" in Apple GarageBand. ${ }^{2}$ As shown in Table 1, the actual number of participants was not even in the all the conditions owing to cases where the access did not reach the end of the experiment.
}

Table 1: Conditions of Experiment1

\begin{tabular}{c|c|c|l|c}
\multicolumn{4}{c}{} & \multicolumn{2}{c|}{ Red } & \multicolumn{2}{l}{ Blue } \\
\cline { 2 - 5 } & Smile & Sad & Smile & Sad \\
\cline { 2 - 5 } High BPM & 39 & 35 & 35 & 29 \\
\hline Low BPM & 32 & 30 & 30 & 35 \\
* Number of participants in each cell
\end{tabular}

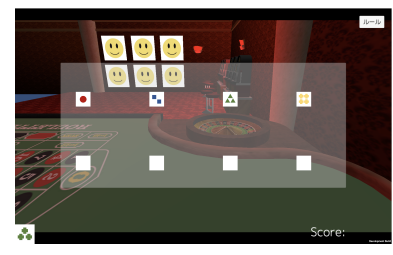

(a) Red and Smile

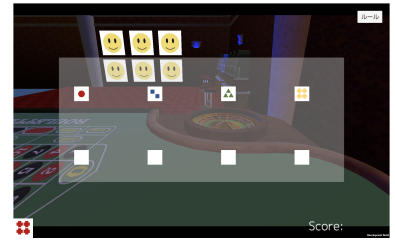

(c) Blue and Smile

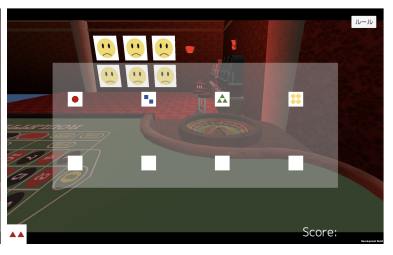

(b) Red and Sad

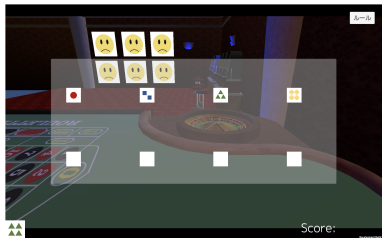

(d) Blue and Sad
Figure 1: Combination of lighting color and expression. The stimuli were presented on background of casino image (a Unity asset (https://unity.com), Common Casino Bundle ๑2014 GreenThumbzNerd.)

4.1.2 Procedure. The participants, recruited via Lancers, read through the instructions for the experimental procedure on the page. Then, they moved to the experimental system on the server by clicking a URL on the page. In the system, the instruction of the game is displayed first. On the instruction page, the participants learned how to operate the system and the rules of WCST. On the screen, following the presentation of the rules, the participants practiced for three turns, and further performed the main task. After completing the task, the participants clicked a button on the screen to access the questionnaire created using Google Forms.

4.1.3 Data. Data were collected using questionnaires and game logs. To test Assumption 1, we asked participants to report their arousal in a questionnaire formatted as a Self-Assessment Manikin (SAM) [4]. The SAM uses illustrations to measure the participants' arousal nonverbally in nine levels, which comprise five illustrations and four items in between. Additionally, the questionnaire included a free description form.

From the game log, the following eight indices were extracted to test Assumption 2:

- Speed

- Time per turn: We evaluated the interval between the placement of a card and the placement of the next card. This is a measure of the speed of decision-making in Assumption 2. 
- Total time: We measured the time from the start to the last turn. This is also an indicator of the speed of decisionmaking in Assumption 2.

- Deliberation

- Reached Category: The ultimate number of changing categories. This is an indicator of the deliberation of decisionmaking in Assumption 2 as it evaluates how well the player responded to the category switch.

- The number of cards required to the first category change (NCRF): This is an indicator of the deliberation in the decision-making process in Assumption 2, as it evaluates the placement of cards in the early stages of the experiment.

- Perseveration error: The number of errors in following the classification of the previous category, even after the category changes. This is an indicator of the deliberation of decision-making in Assumption 2 as it measures the switching between exploration and exploitation in the decision-making. Moreover, in the case there was no category change, the value was set to 30 as the maximum value.

- Number of rule confirmations: The number of times the rule confirmation button was pressed during the game. Since this number varies depending on the participants' understanding of the rules, this is an indicator of the deliberation in decision-making in Assumption 2.

- Performance

- Score: The score obtained in the game. Since this is the outcome of the game, this is an indicator of the performance in the decision-making of Assumption 2.

- Incorrect Answer: The number of cards classified in the incorrectly during the game. Since this is the number of errors in the game, this is a measure of the decision-making performance in Assumption 2.

\subsection{Results}

4.2.1 Means of the Indices. Figure 2 shows the mean values obtained for each condition. Tables 2 and 6 show the results of the 3-way Analysis of Variance (ANOVA). If the ANOVA shows interactions between the factors, the result of the simple main effect is shown in the figure. In the following, we will show the significant results for the questionnaire and the game log.

There was a marginally significant main effect of the color on the arousal $(p=0.06)$. This indicates that the subjective evaluation of arousal increased when the color of the lighting was red rather than blue. This result is consistent with Assumption 1 (effect of color stimuli on arousal).

In the perseveration error, there was a significant main effect of the color $(p=0.03)$ and the facial expression $(p=0.01)$. Figure 2 shows the simple main effect. This result is consistent with Assumption 2 (deliberation). The main effect of the color of the lighting was significant for the number of rule confirmations $(p=0.02)$. This result shows that the number of rule confirmations increases under red lighting and decreases under blue lighting. A marginally significant main effect of color was found regarding incorrect answers $(p=0.09)$. It was suggested that incorrect answers increase under
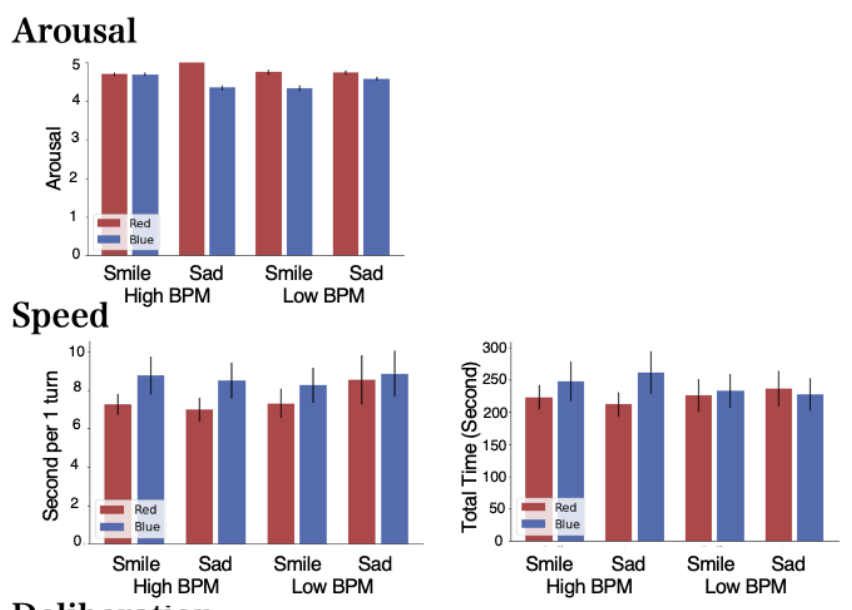

Deliberation
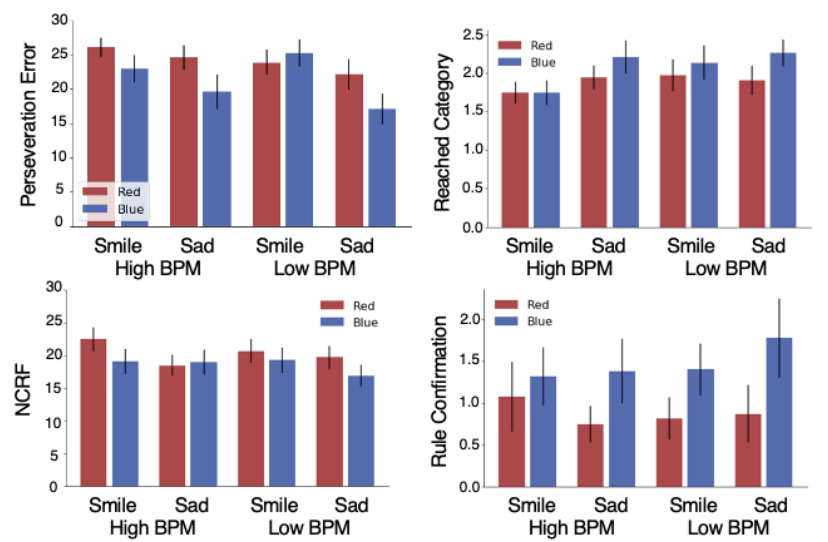

Performance
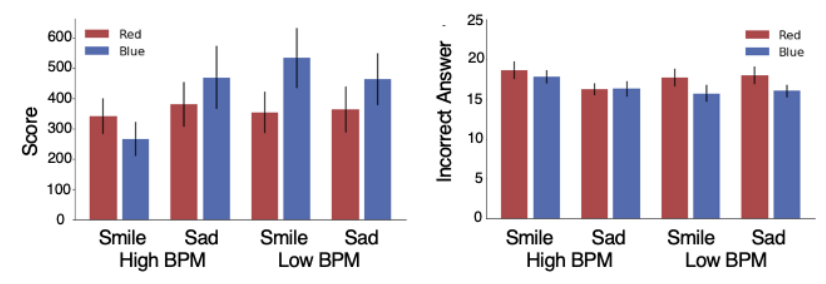

Figure 2: The means and standard errors of Experiment 1

red illumination and decrease under blue illumination. These results are consistent with Assumption 2, the deliberation of decisionmaking and performance in decision-making.

4.2.2 Structural Equation Modeling. We conducted structural equation modeling (SEM) to examine the compatibility with the assumptions. Figure 3 shows the results. This model was constructed to indicate the effect of environmental factors (left) on the performance (right) through arousal (emotion), speed (System 1), and deliberation (System 2) as the internal state-mediating environment, and decision outcome. In this model, the comparative fit index (CFI) was 0.921 , the root mean square error of approximation (RMSEA), and the adjusted goodness of fit index (AGFI) were 0.921, 0.075 , and 0.999 , respectively. This indicates that the model and 
Table 2: F-score in Experiment1

\begin{tabular}{|c|c|c|c|c|c|c|c|c|c|}
\hline & $\begin{array}{l}\overrightarrow{\tilde{W}} \\
\stackrel{0}{0} \\
\dot{Z}\end{array}$ & 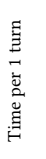 & $\begin{array}{l}\text { 首 } \\
\text { 㞼 } \\
\stackrel{0}{\circ}\end{array}$ & 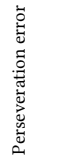 & 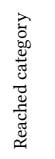 & $\begin{array}{l}\text { 岂 } \\
\text { 乙 }\end{array}$ & 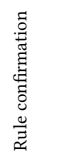 & 苟 & 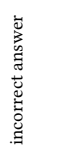 \\
\hline Color (C) & $3.54+$ & 2.70 & 0.99 & $4.50^{*}$ & 2.35 & 1.95 & $5.45^{*}$ & 1.78 & $2.93+$ \\
\hline BGM (B) & 0.74 & 0.32 & 0.09 & 0.77 & 1.48 & 0.26 & 0.11 & 1.37 & 0.30 \\
\hline Expression (E) & 0.48 & 0.25 & 0.01 & $6.95^{* *}$ & 1.97 & 2.17 & 0.02 & 0.69 & 1.41 \\
\hline $\mathrm{C}^{*} \mathrm{~B}$ & 0.33 & 0.46 & 1.05 & 0.65 & 0.25 & 0.09 & 0.37 & 1.47 & 1.29 \\
\hline$B{ }^{*} \mathrm{E}$ & 0.04 & 0.85 & 0.00 & 0.79 & 1.41 & 0.02 & 0.47 & 1.90 & 2.65 \\
\hline$C^{*} \mathrm{E}$ & 0.86 & 0.06 & 0.02 & 2.22 & 0.80 & 0.23 & 0.50 & 0.14 & 0.12 \\
\hline \multirow[t]{2}{*}{$C^{*}{ }^{*}{ }^{*} E$} & 2.25 & 0.06 & 0.32 & 0.70 & 0.02 & 1.11 & 0.01 & 1.24 & 0.12 \\
\hline & & & $+: 1$ & $<0.10$ & $*$ & $<$ & & $*: p$ & $<0.01$ \\
\hline
\end{tabular}

Table 3: Effect size in Experiment 1( $\eta p 2)$

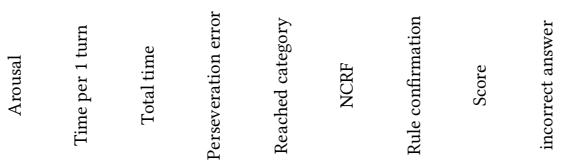

$\begin{array}{llllllllll}\text { Color (C) } & 0.01 & 0.01 & 0.00 & 0.02 & 0.01 & 0.01 & 0.02 & 0.01 & 0.01 \\ \text { BGM (B) } & 0.00 & 0.00 & 0.00 & 0.00 & 0.01 & 0.00 & 0.00 & 0.01 & 0.00 \\ \text { Expression (E) } & 0.00 & 0.00 & 0.00 & 0.03 & 0.01 & 0.01 & 0.00 & 0.00 & 0.01 \\ \text { C * B } & 0.00 & 0.00 & 0.00 & 0.00 & 0.00 & 0.00 & 0.00 & 0.01 & 0.01 \\ \text { B * E } & 0.00 & 0.00 & 0.00 & 0.00 & 0.01 & 0.00 & 0.00 & 0.01 & 0.01 \\ \text { C * E } & 0.00 & 0.00 & 0.00 & 0.00 & 0.00 & 0.00 & 0.00 & 0.00 & 0.00 \\ \text { C * B * E } & 0.00 & 0.00 & 0.00 & 0.00 & 0.00 & 0.00 & 0.00 & 0.00 & 0.00\end{array}$

data fit well. From the path coefficients, which are standardized in the figure, we can confirm that the effect of arousal on speed and deliberation is consistence with Assumption 2.

4.2.3 Additional Analysis of the Perseveration Error. The perseveration error score for those who did not reach the category change was set to 30 in the above analysis. This method is advantageous; it can analyze the effects of the stimulus, including those who did not reach the change in the coping category. However, it does not allow us to measure the degree of change in the flexibility of thinking represented by the persistence error. Therefore, we conducted additional analyses by limiting the number of retention errors to those who reached the category change. First, Figure 4a shows the percentage of people who did and did not reach the first category change. The number of people who reached the category change, except for the Smile condition in the Low-BPM, increased in the blue lighting condition compared with the red lighting condition. Moreover, it is evident that the number of people who reached the category change in the Sad condition increased more than in the Smile condition. We further an conducted the ANOVA on the perseveration error, and excluded those who did not reach the category change (Figure 4). Consequently, we observed a main effect of color $(F(1,76)=12.38$, $p=0.00, \eta p 2=0.15)$, an interaction between color and facial expression $(F(1,76)=3.77, p=0.06, \eta p 2=0.05)$ and a second-order interaction $(F(1,76)=5.49, p=0.02, \eta p 2=0.07)$ in Experiment 1 .

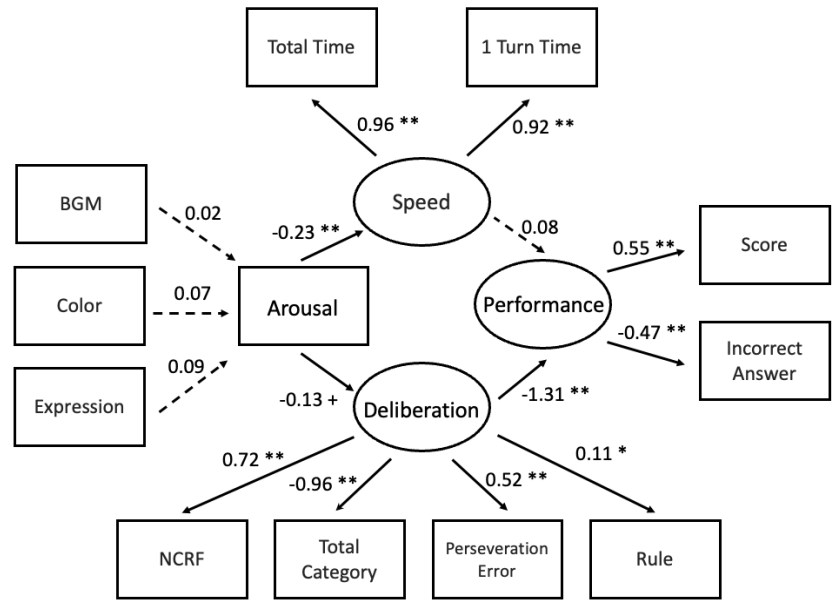

Numbers are Path coefficients. Solid lines: significant, Dashed lines: not significant. $+: p<0.10 \quad *: p<0.05 \quad * *: p<0.01$

Figure 3: SEM of Experiment 1 based on the Assumptions.

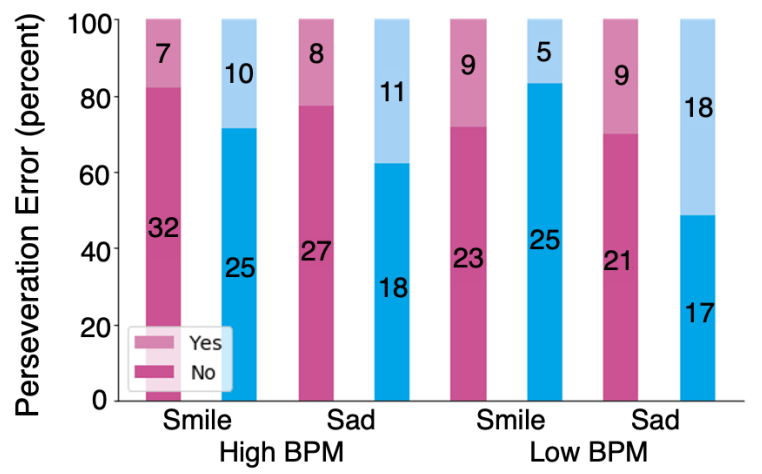

(a) Percentage of participants who reached the first category and those who have did not

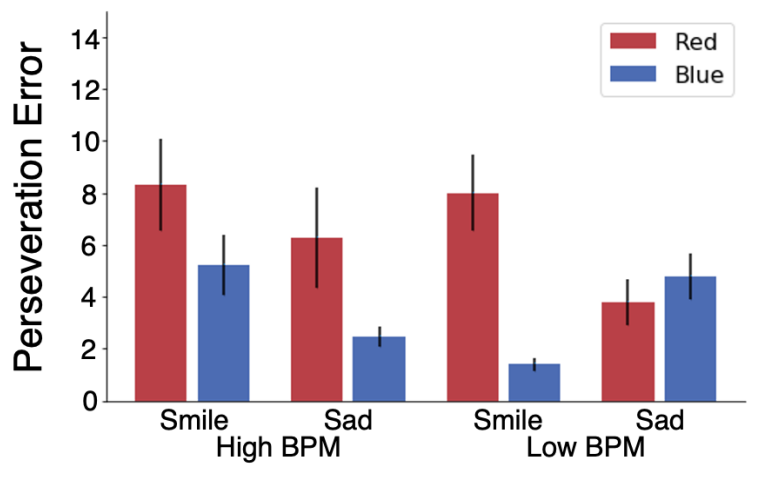

(b) The mean and standard error of perseveration error in experiment 1 without participants who did not reached the first category in Experiment 1

Figure 4: Additional analysis of perseveration error in $\mathbf{E x}-$ periment 1 


\subsection{Discussion}

Experiment 1 shows the effect of the color of the lighting on arousal in the results of the ANOVA. This is consistent with Assumption 1. When the participant's state was System 1, the color of the lighting induced arousal. However, there was no significant difference regarding the BGM. The reason why the BPM of the BGM had no effect on each indicator owing to its volume. In the questionnaire, there were some comments, such as, "The music irritated me" and "The music was too loud." These comments suggest that this factor was involved in the conscious cognitive processes of the participants during the experiment. It is assumed that the induction of emotion by a stimulus is unconsciously effective [3]. This suggests that the attention to the volume may have weakened the effect of the BGM.

The results of the SEM show the correlation assumed in Assumption 2. Furthermore, the perseveration error, the number of rule confirmations, and the number of incorrect answers also changed depending on the effect of the color of the lighting according to the ANOVA results. These may be affected by the arousal as arousal also changes depending on the color of the lighting. There was variation in arousal and decision-making owing to the color of the lighting. However, the path coefficients of the SEM did not show any significant differences. This might be a limitation in evaluating arousal through subjective evaluation.

The additional analysis of the perseveration error shows more complex results with interactions between stimuli, by restricting the analysis to those who reached the category change. The perseveration error was increased by stimuli associated with arousal, such as the red lighting and the smile. However, low-BPM, blue lighting, and sad conditions, which are low-arousal stimuli, increased the errors. It is known that an arousal that is too high or too low reduces cognitive performance $[7,36]$. This law accounts for these results.

\section{EXPERIMENT 2: ENVIRONMENTAL FACTORS AFFECTING SYSTEM 2}

In this experiment, we manipulated the decision-making state of the participants in System 2. Before participating the game, the participants were asked questions to confirm the game rule. Furthermore, we provided additional rewards to the participants who obtained high scores. We expected this to make the participants more focused on the task which would lead to higher scores and fewer incorrect answers than in Experiment 1. Under these conditions, we examined how emotion and decision-making follow these assumptions.

\subsection{Method}

5.1.1 Design. The participants including 239 people recruited via Lancers. There were 123 men and 116 women, with an average age of 41.3 years. They were assigned to the same eight conditions as the participants in Experiment 1. They were further paid 100 yen for their cooperation during the experiment. Additionally, to increase their attention on the task, they received an additional reward of 500 yen if their score was over 1200 points. This score was the top $10 \%$ of the score reached in Experiment 1.
Table 4: Conditions of Experiment2

\begin{tabular}{c|l|l|l|l} 
& \multicolumn{2}{c|}{ Red } & \multicolumn{2}{c}{ Blue } \\
\cline { 2 - 5 } & Smile & Sad & Smile & Sad \\
\cline { 2 - 5 } High BPM & 29 & 25 & 28 & 29 \\
\hline Low BPM & 32 & 34 & 29 & 33 \\
* Number of participants in each cell
\end{tabular}

5.1.2 Procedure. The difference between Experiments 1 and 2 was the presence of the questions. In Experiment 2, the participants were given a confirmation question after reading the rules. The game would not commence until the questions were answered correctly. This procedure made the participants think carefully while playing the game.

\subsection{Results}

5.2.1 Experiment1 vs Experiment2. In the following, we show the significant results for each of the comparisons of the questionnaire and the game log with Experiment 1. By comparing Experiments 1 and 2, differences were observed in four indicators: score, incorrect answers, number of categories reached, and NCRF. The mean score was 358.82 in Experiment 1 and 520.83 in Experiment 2, hence it was higher in Experiment $2(t(503)=3.99, p=0.00)$. The number of incorrect answers decreased in Experiment 2. The mean of these answers were 17.63 in Experiment 1 and 15.19 in Experiment 2 $(t(503)=5.11, p=0.00)$. The means of the number of categories reached first were 1.88 in Experiment 1 and 2.06 in Experiment 2, with an increase in Experiment $2(t(503)=2.06, p=0.03)$. The average number of cards required to reach the first category was 1.88 in Experiment 1 and 2.06 in Experiment 2, with an increase in Experiment $2(t(503)=2.11, p=0.09)$. These indicate that the participants behaved more deliberately in Experiment 2 than they did in Experiment 1. Therefore, we can consider the manipulation in Experiment 2 was successful.

5.2.2 Means of the Indices. Figure 5 shows the mean values obtained for each condition. Tables 5 and 6 show the results of the 3-way ANOVA. If the ANOVA shows that there is an interaction between the factors, a simple main effect is also shown in the figure.

A marginally significant main effect regarding the effect of the icon's facial expression on the arousal $(p=0.07)$ was observed. Participants who were presented with smiley faces showed higher arousal than those who were presented with sad faces. This trend is consistent with Assumption 1 (the effect of emotional contagion on arousal).

The interaction between BGM and facial expressions showed a significant difference in the time per turn $(F(1,231)=3.98, p=$ $0.05, \eta p 2=0.02$ ). The analysis of the simple main effect also showed that participants who were presented with smiley faces spent more time than those who were presented with sad faces in the slow BGM condition. This result was also contrary to Assumption 2 (Speed).

5.2.3 Structural Equation Model. We conducted further SEM for the same purpose as in Experiment 1. We consider that comparing 


\section{Arousal}

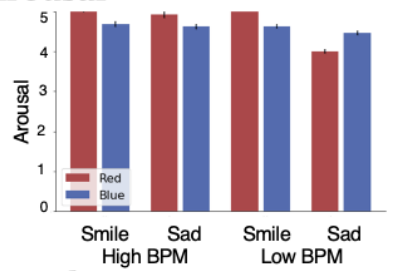

Speed

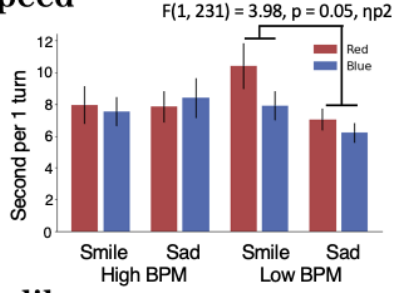

Deliberation

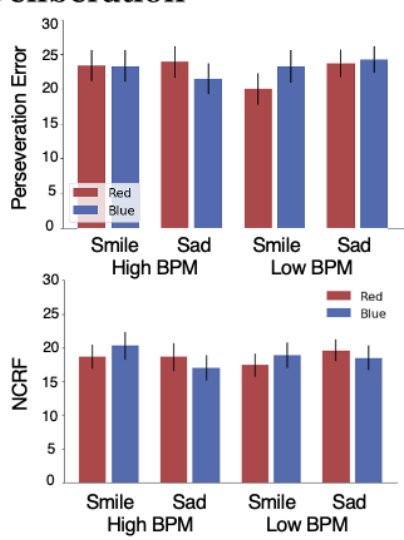

Performance
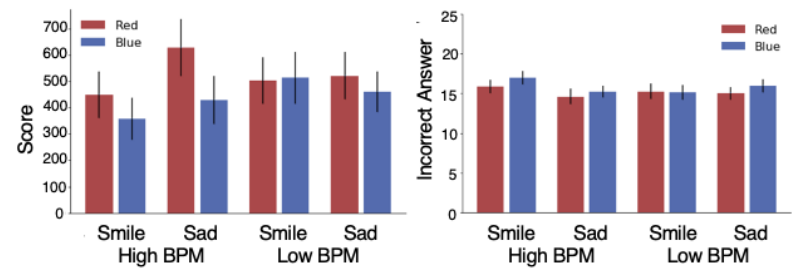

Figure 5: The means and standard errors of Experiment 2

the two SEMs obtained in both the experiments allows us to understand how the internal states affect the relationships between environmental factors, arousal, and decision-making. Figure 6 shows the model where the CFI was 0.988 , the RMSEA was 0.034 , and the AGFI was 1.000. This indicates that the model and data fit well. However, contrary to the model in Experiment 1, this model does not indicate the a significant effect of arousal on speed and deliberation in decision-making.

5.2.4 Additional Analysis of the Perseveration Error. We analyzed the perseveration error only for those who reached the category change, just like in as Experiment 1. Figure 7a shows the percentage of people who reached the category change and those who did not. This figure does not show significant differences between the
Table 5: F-score in Experiment2

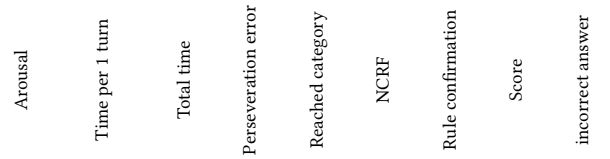

$$
\begin{aligned}
& \begin{array}{llllllllll}
\text { Color (C) } & 1.05 & 1.21 & 1.95 & 0.04 & 0.15 & 0.00 & 0.00 & 1.78 & 1.05
\end{array} \\
& \begin{array}{llllllllll}
\text { BGM (B) } & 0.65 & 0.01 & 0.03 & 0.02 & 0.00 & 0.00 & 0.45 & 0.29 & 0.30
\end{array} \\
& \begin{array}{llllllllll}
\text { Expression (E) } & 3.29+ & 2.18 & 0.98 & 0.32 & 0.42 & 0.09 & 0.12 & 0.70 & 1.05
\end{array} \\
& \begin{array}{llllllllll}
\text { C *B } & 0.11 & 1.46 & 1.94 & 1.03 & 0.25 & 0.00 & 0.01 & 0.90 & 0.14
\end{array}
\end{aligned}
$$

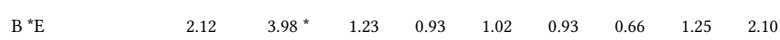

$$
\begin{aligned}
& \begin{array}{llllllllll}
\mathrm{C}{ }^{*} \mathrm{E} & 1.82 & 0.81 & 0.75 & 0.67 & 0.41 & 1.27 & 0.21 & 0.49 & 0.05
\end{array} \\
& \begin{array}{llllllllll}
\mathrm{C}{ }^{*} \mathrm{~B}{ }^{*} \mathrm{E} & 1.52 & 0.06 & 0.71 & 0.00 & 0.01 & 0.02 & 0.58 & 0.02 & 0.39
\end{array} \\
& +: p<0.10 \quad *: p<0.05 \quad * *: p<0.01
\end{aligned}
$$

Table 6: Effect size in Experiment 2 ( $\eta p 2)$

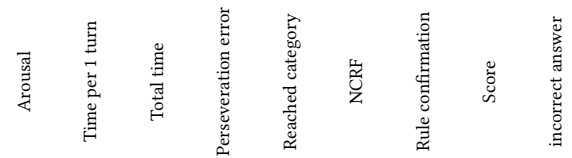$$
\begin{array}{llllllllll}
\text { Color (C) } & 0.00 & 0.01 & 0.01 & 0.00 & 0.00 & 0.00 & 0.00 & 0.01 & 0.00
\end{array}
$$$$
\begin{array}{llllllllll}
\text { BGM (B) } & 0.00 & 0.00 & 0.00 & 0.00 & 0.00 & 0.00 & 0.00 & 0.00 & 0.00
\end{array}
$$$$
\begin{array}{llllllllll}
\text { Expression (E) } \quad 0.01 & 0.01 & 0.00 & 0.00 & 0.00 & 0.00 & 0.00 & 0.00 & 0.00
\end{array}
$$

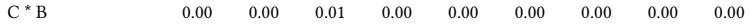$$
\begin{array}{llllllllll}
\mathrm{B}{ }^{*} \mathrm{E} & 0.01 & 0.02 & 0.01 & 0.00 & 0.00 & 0.00 & 0.00 & 0.01 & 0.01
\end{array}
$$

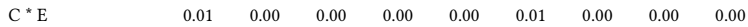$$
\begin{array}{llllllllll}
C^{*}{ }^{*} \mathrm{~B} \text { * E } & 0.01 & 0.00 & 0.00 & 0.00 & 0.00 & 0.00 & 0.00 & 0.00 & 0.00
\end{array}
$$

conditions. We conducted an ANOVA on the participants who reached the category change (Figure 7). Consequently, we observed a main effect of facial expression $(F(1,65)=3.34, p=0.07)$, an interaction between color and facial expression $(F(1,65)=3.48$, $p=0.07)$, and a second-order interaction $(F(1,65)=3.01, p=0.09)$ in Experiment 2.

\subsection{Discussion}

Experiment 2 shows the effect of facial expression on arousal in the ANOVA results. This is consistent with Assumption 1. In a deliberative situation, it is also possible that the facial expression of the icon is more effective in inducing emotion than the color of the lighting.

The results of the SEM show no influence of arousal on decisionmaking. Given this result, we can conclude that arousal does not affect behavior during the task when participants are at a sufficiently high arousal level (System 2). Therefore, we found an exception to Assumption 2, by focusing on the internal factors of decision-making. Moreover, in Experiment 2, there was less change in decision-making by condition than in Experiment 1. It is considered that emotions have an insignificant influence on decisionmaking in System 2.

The additional analysis shows that the perseveration error tends to increase in the sad condition. This seems contradict the results of Experiment 1 . The result can be interpreted as the calming stimulus 


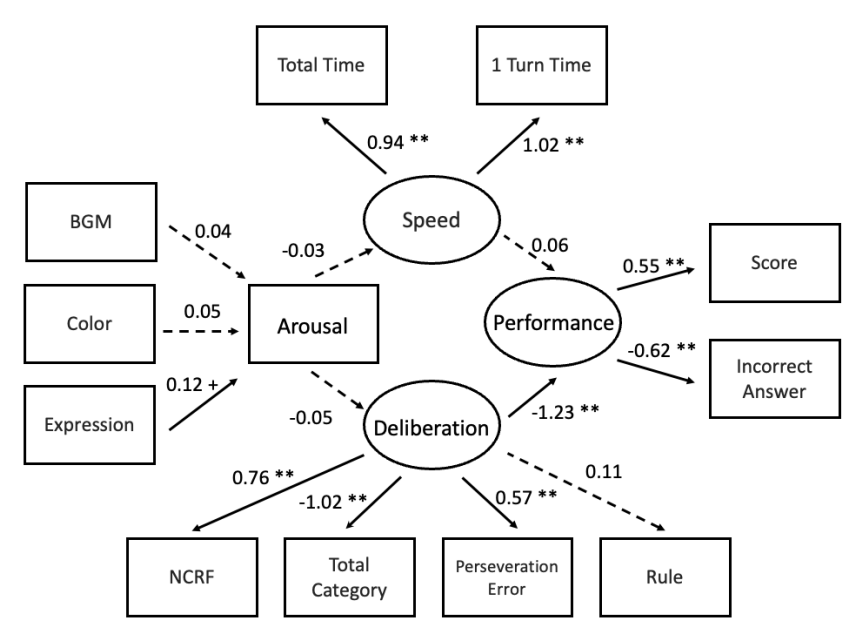

Numbers are Path coefficient. Solid lines: significant, Dashed lines: no significant. $+: p<0.10 \quad *: p<0.05 \quad * *: p<0.01$

Figure 6: SEM of Experiment 2 based on the Assumptions.

increasing errors when high arousal was sufficiently evoked. This interpretation is also supported by the result of the time spent per turn, which decreased in the sad condition. The effects of facial expressions vary depending on the context [11]. Additionally, the red smiles at Low BPM are likely to be erroneous in the secondorder interaction. This condition also resulted in many mistakes in Experiment 1. It is possible that the red smile stimulus itself induces errors.

\section{GENERAL DISCUSSION AND CONCLUSION}

In this study, we examined the interactions between internal and external factors by manipulating the color of the lighting, the BGM, and the icon's facial expressions given different internal states.

As a result of Experiment 1, in which the participants were under the System 1 process, we found that the color of the lighting influenced arousal, which further affected decision-making. Specifically, there were differences in the number of rule confirmations, incorrect answers, and perseveration errors. These results are consistent with Assumptions 1 and 2. In contrast, in Experiment 2, where the participants were under the System 2, the effect of arousal on the icon's facial expressions was suggested. This result is in line with Assumption 1. In this experiment, we observed the opposite results for Assumption 2. Moreover, there were fewer changes in the decision-making based on arousal in Experiment 2 than in Experiment 1.

Additionally, a comparison of Experiments 1 and 2 confirmed the influence of more stimuli on decision-making in Experiment 1. There was also a difference in how decision-making changed between the two experiments. Moreover, it is worth noting that the effect of arousal on decision-making was significant in the SEM of Experiment 1, rather than in the SEM of Experiment 2. The results show that users in System 2 might not have the necessary support from affective interfaces. Thus, it was found that the influence of

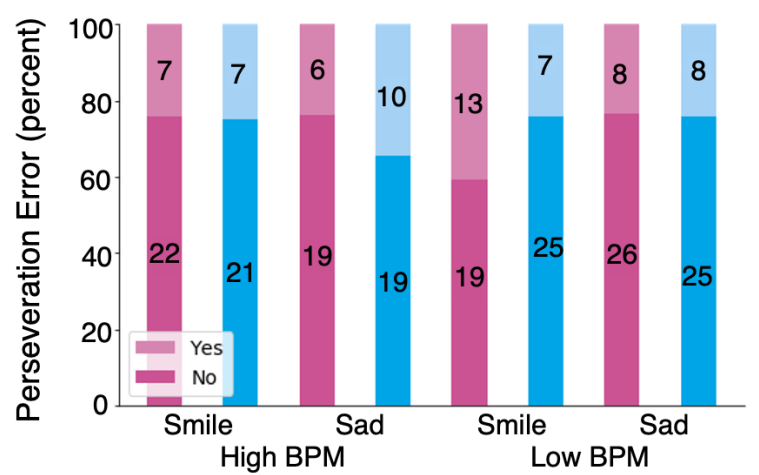

(a) Percentage of participants who reached the first category and those who did not in Experiment 2

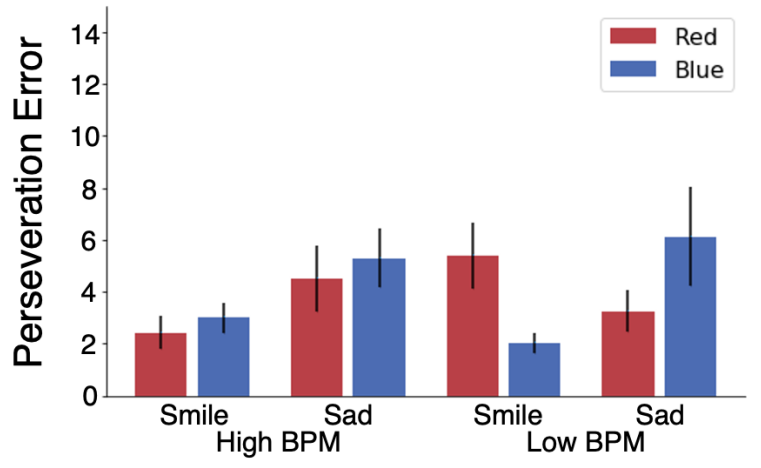

(b) The means and standard error of perseveration errors in Experiment 2 without participants who did not reached the first category

Figure 7: Additional analysis of the perseveration error in Experiment 2

the external environment on emotion and decision-making differed depending on the participant's internal state.

This study has several limitations. For example, because arousal was evaluated through subjective evaluation, it might not completely reflect the internal state of the participants. Therefore, it is necessary to conduct experiments using physiological data in the future. Moreover, it is necessary to conduct a laboratory experiment to examine the detailed decision-making process.

Through such explorations of the basic mechanism between emotional states and decision-making, it will be possible to develop an agent-based support for better decision making. We consider findings in this study especially beneficial for an agent-based system using ambient technologies, where the facial expressions of agents surrounding the user induce a specific mood [31, 35].

\section{ACKNOWLEDGMENTS}

This work was supported by JSPS KAKENHI Grant Number $20 \mathrm{H} 05560$.

\section{REFERENCES}

[1] Adam R Aron, Stephen Monsell, Barbara J Sahakian, and Trevor W Robbins. 2004. A componential analysis of task-switching deficits associated with lesions of left and right frontal cortex. Brain 127, 7 (2004), 1561-1573. https://doi.org/10.1093/ brain/awh169 
[2] Sigal G Barsade. 2002. The ripple effect: Emotional contagion and its influence on group behavior. Administrative Science Quarterly 47, 4 (2002), 644-675. https: //doi.org/10.2307/3094912

[3] Kent Berridge and Piotr Winkielman. 2003. What is an unconscious emotion? (The case for unconscious "liking"). Cognition and Emotion 17, 2 (2003), 181-211. https://doi.org/10.1080/02699930302289

[4] Margaret M Bradley and Peter J Lang. 1994. Measuring emotion: the selfassessment manikin and the semantic differential. Fournal of Behavior Therapy and Experimental Psychiatry 25, 1 (1994), 49-59. https://doi.org/10.1016/00057916(94)90063-9

[5] Rodney Brooks. 1986. A robust layered control system for a mobile robot. IEEE Journal on Robotics and Automation 2, 1 (1986), 14-23. https://doi.org/10.1109/ JRA.1986.1087032

[6] Jean Costa, François Guimbretière, Malte F Jung, and Tanzeem Choudhury. 2019 Boostmeup: Improving cognitive performance in the moment by unobtrusively regulating emotions with a smartwatch. Proceedings of the ACM on Interactive, Mobile, Wearable and Ubiquitous Technologies 3, 2 (2019), 1-23. https://doi.org/ $10.1145 / 3328911$

[7] Mihaly Csikszentmihalyi. 1990. Flow: The psychology of optimal experience. Harper \& Row New York

[8] Antonio Damasio. 1999. The feeling of what happens: Body and emotion in the making of consciousness. Harcourt College Publishers.

[9] Antonio R Damasio. 1996. The somatic marker hypothesis and the possible functions of the prefrontal cortex. Philosophical Transactions of the Royal Society of London. Series B: Biological Sciences 351, 1346 (1996), 1413-1420. https://doi org $/ 10.1098 / \mathrm{rstb} .1996 .0125$

[10] Wim De Neys. 2018. Dual process theory 2.0. Routledge.

[11] Paul Ekman and Wallace V Friesen. 1982. Felt, false, and miserable smiles. fournal of Nonverbal Behavior 6, 4 (1982), 238-252. https://doi.org/10.1007/BF00987191

[12] Paul Eling, Kristianne Derckx, and Roald Maes. 2008. On the historical and conceptual background of the Wisconsin Card Sorting Test. Brain and Cognition 67, 3 (2008), 247-253. https://doi.org/10.1016/j.bandc.2008.01.006

[13] Jonathan St BT Evans and Keith E Stanovich. 2013. Dual-process theories of higher cognition: Advancing the debate. Perspectives on Psychological Science 8, 3 (2013), 223-241. https://doi.org/10.1177/1745691612460685

[14] Alicia Fernández-Sotos, Antonio Fernández-Caballero, and José M Latorre. 2016. Influence of tempo and rhythmic unit in musical emotion regulation. Frontiers in Computational Neuroscience 10 (2016), 80. https://doi.org/10.3389/fncom.2016. 00080

[15] James M Gold, Constance Carpenter, Christopher Randolph, Terry E Goldberg and Daniel R Weinberger. 1997. Auditory working memory and Wisconsin Card Sorting Test performance in schizophrenia. Archives of General Psychiatry 54, 2 (1997), 159-165. https://doi.org/10.1001/archpsyc.1997.01830140071013

[16] Joseph K Goodman, Cynthia E Cryder, and Amar Cheema. 2013. Data collection in a flat world: The strengths and weaknesses of Mechanical Turk samples. Fournal of Behavioral Decision Making 26, 3 (2013), 213-224. https://doi.org/10.1002/bdm. 1753

[17] Stefan G Hofmann, Sanna Heering, Alice T Sawyer, and Anu Asnaani. 2009. How to handle anxiety: The effects of reappraisal, acceptance, and suppression strategies on anxious arousal. Behaviour Research and Therapy 47, 5 (2009), 389-394. https://doi.org/10.1016/j.brat.2009.02.010

[18] Jon Kabat-Zinn and Thich Nhat Hanh. 2009. Full catastrophe living: Using the wisdom of your body and mind to face stress, pain, and illness. Delta.

[19] Daniel Kahneman. 2011. Thinking, Fast and Slow. Farrar, Straus and Giroux, New York.

[20] S. Konishi, M. Kawazu, I. Uchida, H. Kikyo, I. Asakura, and Y. Miyashita. 1999 Contribution of working memory to transient activation in human inferior prefrontal cortex during performance of the Wisconsin Card Sorting Test. Cerebral Cortex 9 (1999), 45-53. Issue 7. https://doi.org/10.1093/cercor/9.7.745

[21] Rikard Küller, Byron Mikellides, and Jan Janssens. 2009. Color, arousal, and performance-A comparison of three experiments. Color Research \& Application 34, 2 (2009), 141-152. https://doi.org/10.1002/col.20476

[22] M Laiacona, MG Inzaghi, A De Tanti, and E Capitani. 2000. Wisconsin card sorting test: a new global score, with Italian norms, and its relationship with the Weigl sorting test. Neurological Sciences 21, 5 (2000), 279-291. https://doi.org/10. 1007/s100720070065

[23] Grace Leslie, Asma Ghandeharioun, Diane Zhou, and Rosalind W Picard. 2019. Engineering Music to Slow Breathing and Invite Relaxed Physiology. In 2019 8th International Conference on Affective Computing and Intelligent Interaction (ACII). IEEE, IEEE Computer Society, Los Alamitos, CA, USA, 1-7. https://doi.org/10. 1109/ACII.2019.8925531

[24] Chuh-Hyoun Lie, Karsten Specht, John C Marshall, and Gereon R Fink. 2006. Using fMRI to decompose the neural processes underlying the Wisconsin Card Sorting Test. Neuroimage 30, 3 (2006), 1038-1049. https://doi.org/10.1016/j. neuroimage.2005.10.031

[25] Sonia J Lupien, Christian J Gillin, and Richard L Hauger. 1999. Working memory is more sensitive than declarative memory to the acute effects of corticosteroids: A dose-response study in humans. Behavioral Neuroscience 113, 3 (1999), 420. https://doi.org/10.1037//0735-7044.113.3.420

[26] Wim De Neys. 2006. Dual processing in reasoning: Two systems but one reasoner. Psychological Science 17, 5 (2006), 428-433. https://doi.org/10.1111/j.1467-9280. 2006.01723.x

[27] Hideki Ohira, Masahiro Matsunaga, Hiroki Murakami, Takahiro Osumi, Seisuke Fukuyama, Jun Shinoda, and Jitsuhiro Yamada. 2013. Neural mechanisms mediating association of sympathetic activity and exploration in decision-making. Neuroscience 246 (2013), 362-374. https://doi.org/10.1016/j.neuroscience.2013.04.050

[28] Raul Benites Paradeda, Carlos Martinho, and Ana Paiva. 2020. Persuasion Strategies Using a Social Robot in an Interactive Storytelling Scenario. In Proceedings of the 8th International Conference on Human-Agent Interaction (Virtual Event, USA) (HAI '20). Association for Computing Machinery, New York, NY, USA, 69-77. https://doi.org/10.1145/3406499.3415084

[29] Gordon Pennycook, Jonathan A Fugelsang, and Derek J Koehler. 2015. What makes us think? A three-stage dual-process model of analytic engagement. Cognitive Psychology 80 (2015), 34-72. https://doi.org/10.1016/j.cogpsych.2015.05.001

[30] James A Russell. 1980. A circumplex model of affect. fournal of Personality and Social Psychology 39, 6 (1980), 1161. https://doi.org/10.1037/h0077714

[31] Elaheh Sanoubari, Denise Y. Geiskkovitch, Diljot S. Garcha, Shahed A. Sabab, Kenny Hong, James E. Young, Andrea Bunt, and Pourang Irani. 2018. Subliminal Priming in Human-Agent Interaction: Can Agents Use Single-Frame Visuals in Video Feeds to Shape User Perceptions?. In Proceedings of the 6th International Conference on Human-Agent Interaction (Southampton, United Kingdom) (HAI '18). Association for Computing Machinery, New York, NY, USA, 205-213. https: //doi.org/10.1145/3284432.3284447

[32] Daniela Schoofs, Oliver T Wolf, and Tom Smeets. 2009. Cold pressor stress impairs performance on working memory tasks requiring executive functions in healthy young men. Behavioral Neuroscience 123, 5 (2009), 1066. https: //doi.org/10.1037/a0016980

[33] Jaime Snyder, Mark Matthews, Jacqueline Chien, Pamara F Chang, Emily Sun, Saeed Abdullah, and Geri Gay. 2015. Moodlight: Exploring personal and social implications of ambient display of biosensor data. In Proceedings of the 18th ACM Conference on Computer Supported Cooperative Work \& Social Computing. Association for Computing Machinery, New York, NY, USA, 143-153. https: //doi.org/10.1145/2675133.2675191

[34] Jean Walters, Michael J Apter, and Sven Svebak. 1982. Color preference, arousal, and the theory of psychological reversals. Motivation and Emotion 6, 3 (1982), 193-215. https://doi.org/10.1007/BF00992245

[35] Cong Wang, Xin Wan, Naoto Yoshida, and Tomoko Yonezawa. 2020. Stimulation of Motivation for Learning by Multiple Agents in Group Training. In Proceedings of the 8th International Conference on Human-Agent Interaction (Virtual Event, USA) (HAI '20). Association for Computing Machinery, New York, NY, USA, 25-31. https://doi.org/10.1145/3406499.3415069

[36] Robert M Yerkes, John D Dodson, et al. 1908. The relation of strength of stimulus to rapidity of habit-formation. Fournal of Comparative Neurology and Psychology 18 (1908), 459-482. https://doi.org/10.1002/cne.920180503

[37] Shigeo Yoshida, Tomohiro Tanikawa, Sho Sakurai, Michitaka Hirose, and Takuji Narumi. 2013. Manipulation of an emotional experience by real-time deformed facial feedback. In Proceedings of the 4th Augmented Human International Conference (Stuttgart, Germany) (AH '13). Association for Computing Machinery, New York, NY, USA, 35-42. https://doi.org/10.1145/2459236.2459243 